\title{
An incremental micromechanical scheme for nonlinear particulate composites
}

\author{
L.H. Dai*, G.J. Huang \\ State Key Laboratory of Nonlinear Mechanics (LNM), Institute of Mechanics, Chinese Academy of Sciences, \\ Beijing 100080, People's Republic of China
}

Received 10 August 1999; received in revised form 5 July 2000

\begin{abstract}
A general incremental micromechanical scheme for the nonlinear behavior of particulate composites is presented in this paper. The advantage of this scheme is that it can reflect partly the effects of the third invariant of the stress on the overall mechanical behavior of nonlinear composites. The difficulty involved is the determination of the effective compliance tensors of the anisotropic multiphase composites. This is completed by making use of the generalized self-consistent Mori-Tanaka method which was recently developed by Dai et al. (Polymer Composites 19 (1998) 506-513; Acta Mechanica Solida 18 (1998) 199-208). Comparison with existing theoretical and numerical results demonstrates that the present incremental scheme is quite satisfactory. Based on this incremental scheme, the overall mechanical behavior of a hardparticle reinforced metal matrix composite with progressive particle debonding damage is investigated. (C) 2001 Elsevier Science Ltd. All rights reserved.
\end{abstract}

Keywords: Incremental method; Composites; Micromechanics; Nonlinear behavior

\section{Introduction}

It is well known that most widely used metal matrix composites and polymer matrix composites behave in the nonlinear range under mechanical loadings. Therefore, theoretical prediction of the overall mechanical behavior of this class of nonlinear composites is important. However, due to the difficulty in solving nonlinear boundary value problems, exact solutions by analytical methods

\footnotetext{
* Corresponding author.

E-mail address: 1hdai@Inm.imech.ac.cn (L.H. Dai).
} 
cannot be obtained except for some special cases, such as composites with regular microstructure [1]. Instead of finding the exact solutions, several powerful approximate or linearized approaches have been developed to estimate or bound the overall properties of nonlinear composite materials during the past two decades. Among the most typical approaches are the secant modulus method, the nonlinear variational approach, and the simplified incremental method. Based on the idea of using the linear effective properties of a comparison composite to estimate its nonlinear properties, Tandon and Weng [2] proposed the so-called classical secant modulus method (CSMM) to analyze the plastic behavior of particulate composites. Their idea evolved out of the earlier work on polycrystal plasticity $[3,4]$. Since the von Mises equivalent stress of the matrix was calculated directly from its mean deviatoric stress, CSMM cannot predict the plastic response of porous materials subjected to a pure hydrostatic loading. To eliminate this drawback, Qiu and Weng [5,6] and $\mathrm{Hu}$ [7] developed the modified secant modulus method (MSMM) which allows one to evaluate the equivalent stress of the matrix by making use of the second moment of the matrix deviatoric stress. Ju and Tseng [8] proposed a new approach called the matrix stress norm to evaluate the equivalent stress of the nonlinear matrix. Another powerful tool to estimate or bound the overall behavior of nonlinear composites is the nonlinear variational method $[9,10]$. Recently, $\mathrm{Hu}$ [7] demonstrated that the use of the exact second moment of the equivalent stress in the context of MSMM leads to the same results as the nonlinear variational method. Recently, a powerful incremental damage theory was developed by Tohgo et al. [11-13] by making use of Eshelby's equivalent inclusion concept [14] and the Mori-Tanaka mean-field theory [15].

It is noted that, although these homogenization methods are concise in concept and convenient in operation, they still suffer the limitation that they cannot reflect the effect of the third stress invariant on the overall behavior of composites. However, recent research work by Lee and Mear [16] has demonstrated that the effect of the third stress invariant on the overall response cannot be ignored for some power-law materials reinforced by rigid particles. Therefore, some new approaches which can remove, at least partly, this limitation need to be developed for predicting accurately the overall behavior of nonlinear composites.

According to the aforementioned observations, a general incremental scheme is presented in this paper. This scheme involves the instantaneous anisotropic tangent modulus tensors of nonlinear phases thus allowing one to consider partly the effects of the third stress invariant on the overall behavior of nonlinear composites. The validity of the present incremental scheme is checked by comparing it with the available micromechanical methods.

\section{Effective compliance of anisotropic composites}

Since the incremental scheme involves the anisotropic tangent compliance tensor of the phases and the composite, we present a short description of their determination by extending the generalized self-consistent Mori-Tanaka method (GSCMTM) developed initially for isotropic composites by Dai et al. [17,18] to an anisotropic case in this section.

Consider a two-phase anisotropic composite material with inclusions distributed uniformly in a matrix. Inspired by the idea of the generalized self-consistent method (GSCM) [19], the composite can be modeled by a three-phase geometric configuration: inclusion/matrix/effective composite medium in order to investigate the effective moduli or compliance of the composite. The 
elastic modulus tensors of the matrix, the inclusion, and the composite are denoted, respectively, by $\mathbf{L}^{(0)}, \mathbf{L}^{(1)}, \overline{\mathbf{L}}$, and the corresponding compliance tensors can be obtained by the relation $\mathbf{M}=\mathbf{L}^{-1}$. When the homogeneous displacement boundary condition $\left.u\right|_{\partial V}=\mathbf{E} \cdot x$ is imposed at infinity, the strain at an arbitrary point in the composite can be determined by the following Lippman-Schinger-Dyson-type integral equation [20]:

$$
\boldsymbol{\varepsilon}(x)=\mathbf{E}-\int_{V} \boldsymbol{\Gamma}\left(x-x^{\prime}\right): \delta \mathbf{L}: \varepsilon\left(x^{\prime}\right) \mathrm{d} x
$$

where $\delta \mathbf{L}$ is the fluctuating part of the elastic moduli tensor of the composite and defined by: $\delta \mathbf{L}(x)=\mathbf{L}(x)-\overline{\mathbf{L}}$, where $\mathbf{L}(x)$ is the local elastic moduli tensor of the composite. $\boldsymbol{\Gamma}\left(x-x^{\prime}\right)$ is the modified Green function associated with the homogeneous comparison material. By taking the effective composite medium as the comparison material and making use of the strain equivalent condition of GSCM and Hill's interfacial operation theory [21], one can derive the effective moduli of anisotropic composites as follows [22]:

$$
\overline{\mathbf{L}}=\mathbf{L}^{(0)}+c_{1}\left(\mathbf{L}^{(1)}-\mathbf{L}^{(0)}\right):\left[c_{1} \stackrel{(4)}{\mathbf{I}}+\left(1-c_{1}\right) \mathbf{H}\right]^{-1} .
$$

Similarly, the effective compliance of the composite can be easily obtained:

$$
\overline{\mathbf{M}}=\mathbf{M}^{(0)}+c_{1}\left(\mathbf{M}^{(1)}-\mathbf{M}^{(0)}\right):\left[c_{0} \mathbf{M}^{(0)^{-1}}: \mathbf{H}: \mathbf{M}^{(1)}+c_{1} \mathbf{I}\right]^{-1},
$$

(4)

where $c_{0}, c_{1}$ are the volume fraction of matrix and inclusion, respectively, $\mathbf{I}$ is the fourth rank unit tensor. Likewise, $\mathbf{H}$ is defined as

$$
\mathbf{H}=\stackrel{(4)}{\mathbf{I}}+\mathbf{P}:\left(\mathbf{M}^{(1)^{-1}}-\mathbf{M}^{(0)^{-1}}\right),
$$

where $\mathbf{P}$ is related to the Eshelby tensor of inclusion $\mathbf{S}$ by $\mathbf{P}=\mathbf{S}: \mathbf{M}^{(0)}$ and $\mathbf{S}$ is defined by Eq. (12). It is interesting to find that the derived result of Eq. (2) or Eq. (3) is identical to that of the Mori-Tanaka method (MTM) [23]. Since the method starts from GSCM and leads to the same result of MTM, we call our present method as the generalized self-consistent Mori-Tanaka method (GSCMTM). The advantages of GSCMTM are that it can be suitable for arbitrary shape inclusion and anisotropic phases cases. Furthermore, in comparison with MTM, GSCMTM can easily be extended to the coated inclusion-based composites [18].

\section{Incremental scheme for two-phase nonlinear composites}

Consider inclusion/matrix two-phase nonlinear composites. The local constitutive behavior of individual phases can be characterized by a strain potential $U(\boldsymbol{\sigma})$

$$
\varepsilon=\frac{\partial U(\boldsymbol{\sigma})}{\partial \boldsymbol{\sigma}}
$$


This constitutive model is commonly used to represent a number of nonlinear mechanical phenomena, including time-independent plastic deformation (i.e. deformation theory of plasticity) and time-dependent viscous deformation (e.g. high-temperature creep) of metals. In the first case, $\boldsymbol{\sigma}$ and $\boldsymbol{\varepsilon}$ are the infinitesimal stress and strain tensors. In the second case, $\boldsymbol{\sigma}$ and $\boldsymbol{\varepsilon}$ should be identified with the Cauchy stress and Eulerian strain rate, respectively. For simplicity, attention is focused on a class of isotropic materials for which $U(\boldsymbol{\sigma})$ reads as

$$
U^{(r)}(\boldsymbol{\sigma})=\frac{1}{2 k^{(r)}} \sigma_{m}^{2}+\varphi^{(r)}\left(\sigma_{e}\right) \quad(r=0,1),
$$

where $\sigma_{m}=\operatorname{tr}(\boldsymbol{\sigma}) / 3$ and $\sigma_{e}=(3 \mathbf{s}: \mathbf{s} / 2)^{1 / 2}$ are the first two invariants of $\boldsymbol{\sigma}$, while $\mathbf{s}$ is the deviatoric stress. By differentiation with respect to time, the constitutive relation (5) can be expressed in the incremental form

$$
\dot{\boldsymbol{\varepsilon}}=\mathbf{M}_{t}(\boldsymbol{\sigma}): \dot{\boldsymbol{\sigma}},
$$

$\mathbf{M}_{t}$ is the instantaneous tangent compliance tensor and can be written as

$$
\mathbf{M}_{t}(\boldsymbol{\sigma})=\frac{1}{3 k} \mathbf{J}+\frac{1}{2 \mu_{t}} \mathbf{K}+\frac{1}{\lambda} \mathbf{F},
$$

where

$$
\begin{aligned}
& \mathbf{J}=\stackrel{(2)}{\mathbf{I}} \otimes \stackrel{(2)}{\mathbf{I}} / 3, \quad \mathbf{K}=\stackrel{(4)}{\mathbf{I}}-\stackrel{(2)}{\mathbf{I}} \otimes \stackrel{(2)}{\mathbf{I}} / 3, \\
& \mathbf{F}=\widehat{\mathbf{s}} \otimes \widehat{\mathbf{s}}, \quad \widehat{\mathbf{s}} \equiv \mathbf{s} / \sigma_{e}
\end{aligned}
$$

and

$$
\begin{aligned}
& k=\sigma_{m} /[3 \partial U(\boldsymbol{\sigma}) / \partial \operatorname{tr}(\boldsymbol{\sigma})], \quad \mu_{t}=\sigma_{e} / 3 \varphi^{\prime}\left(\sigma_{e}\right), \\
& \lambda=\left(\frac{4}{9}\right)\left[\sigma_{e} /\left(\sigma_{e} \varphi^{\prime \prime}\left(\sigma_{e}\right)-\varphi^{\prime}\left(\sigma_{e}\right)\right],\right. \\
& \varphi^{\prime}\left(\sigma_{e}\right)=\partial \varphi\left(\sigma_{e}\right) / \partial \sigma_{e} .
\end{aligned}
$$

In a general case, the local tangent compliance of the phases is anisotropic. The tangent compliance varying from one point to another, an approximation is made and constant tangent tensors are considered in each phase. It is noted that the tangent compliance defined by Eq. (8) depends not only on the first two stress invariants $\left(\sigma_{m}, \sigma_{e}\right)$ but also on $\widehat{\mathbf{s}} \otimes \widehat{\mathbf{s}}$. While $\widehat{\mathbf{s}} \otimes \widehat{\mathbf{s}}$ cannot be completely determined by the first two stress invariants, it also depends on the third stress invariant. Hence, the presently defined tangent compliance can, at least partly reflect the effect of the third invariant of stress. It is this point that differs from the existing methods, such as the secant modulus method [2,4-7] and the incremental method where the isotropic tangent modulus tensors are adopted [11-13].

The overall behavior of nonlinear composites can be characterized by the following incremental constitutive relation:

$$
\dot{\mathbf{E}}=\overline{\mathbf{M}}_{t}(\boldsymbol{\Sigma}): \dot{\Sigma},
$$


where $\mathbf{E}, \boldsymbol{\Sigma}$ are the macroscopic strain and stress tensors, respectively. Usually, determining the anisotropic effective compliance $\overline{\mathbf{M}}_{t}$ is very difficult due to the anisotropic matrix inclusion problem which has to be solved. In our present approach, $\overline{\mathbf{M}}_{t}$ will be determined by GSCMTM. The involved anisotropic Eshelby tensor $\mathbf{S}$ was given by Mura [24]:

$$
S_{i j k l}=(1 / 8 \pi) M_{m n k l}^{(0)}\left(\bar{G}_{i m j n}+\bar{G}_{j \mathrm{~min}}\right),
$$

where

$$
\bar{G}_{i j k l}=2 \pi \int_{-1}^{1} R\left[G_{i j k l} / Z\right] \mathrm{d} \bar{\xi}_{3},
$$

in which $R\left[G_{i j k l} / Z\right]$ is the sum of the residues of the function $G_{i j k l} / Z$ existing within the unit circle $|Z|=1$ under fixed value of $\bar{\xi}_{3}$, while $G_{i j k l}$ is defined by

$$
G_{i j k l}=\bar{\xi}_{k} \bar{\xi}_{l} N_{i j}(\bar{\xi}) / D(\bar{\xi}),
$$

where $N_{i j}(\bar{\xi}), D(\bar{\xi})$ are the cofactors and the determinant of Christoffel matrix. The closed form of solutions of $\bar{G}_{i j k l}$ can only be obtained for orthotropic and transversely isotropic materials. According to this fact, we only consider uniaxial tension and axisymmetric tension loading cases which ensure that the tangent compliance is transversely isotropic.

The overall behavior of nonlinear composites can be modeled by a series of incremental steps. In each incremental step, the nonlinear composite will be characterized approximately by a linear one. In this paper we only consider the case where the matrix phase is nonlinear while the spherical inclusion phase remains elastic during the entire deformation procedure. Because the tangent compliance depends on the instantaneous stress state, the average stresses of the matrix in each step should be determined. If the average stresses in the last step are known, then the average stresses of the matrix in the present step can be obtained by the approach to linear composites [22]:

$$
\begin{aligned}
\mathrm{d} \bar{\sigma}_{m}^{(0)} & =\frac{1}{\left(1-c_{1}\right)+c_{1}\left(k^{(1)} / k^{(0)}\right) h_{k}} \mathrm{~d} \Sigma_{m}, \\
\mathrm{~d} \bar{\sigma}_{e}^{(0)} & =\frac{1}{\left(1-c_{1}\right)+c_{1}\left(\mu^{(1)} / \mu_{t}^{(0)}\right) h_{\mu}} \mathrm{d} \Sigma_{e},
\end{aligned}
$$

where

$$
\begin{aligned}
& h_{k}=\frac{3 k^{(1)}+4 \mu_{t}^{(0)}}{3 k^{(0)}+4 \mu_{t}^{(0)}} \\
& h_{\mu}=1+\frac{6}{5} \frac{\left(\mu^{(1)}-\mu_{t}^{(0)}\right)\left(k^{(0)}+2 \mu_{t}^{(0)}\right)}{\mu_{t}^{(0)}\left(3 k^{(0)}+4 \mu_{t}^{(0)}\right)},
\end{aligned}
$$

where $k$ and $\mu$ are bulk and shear moduli, respectively, and $\Sigma_{m}$ and $\Sigma_{e}$ are the first two stress invariants of the imposed stress tensor $\boldsymbol{\Sigma}$. Once the local tangent compliance of the phases is obtained, the overall tangent compliance of the composite can be determined by Eq. (3) for each incremental step. Therefore, the overall stress-strain curve can be obtained point by point by making use of the incremental constitutive relation of Eq. (7). 
Consider uniaxial tension: $\Sigma_{11}=\Sigma_{22}=0, \Sigma_{33} \neq 0$. For this case, the tangent compliance tensor and the other related tensor are transversely isotropic tensors which can be expressed and calculated by a set of rules given by Walpole [25]. The overall stress-strain relation of nonlinear composites can be written as

$$
\dot{E}_{33}=\bar{M}_{3333}^{t} \dot{\Sigma}_{3333}
$$

$\bar{M}_{3333}^{t}$ is the overall longitudinal tangent compliance tensor of the composite and is given by

$$
\bar{M}_{3333}^{t}=M_{3333}^{(0) t}+c_{1} \frac{y_{1}}{y_{1} y_{4}-2 y_{2} y_{3}},
$$

where

$$
\begin{aligned}
& y_{1}=\left[2\left(k^{(1)}+\mu^{(1)} / 3\right) / w_{1}-M_{3333}^{(0) t}\right] / w_{2}+c_{0} q_{1}, \\
& y_{2}=\left[-\left(k^{(1)}-2 \mu^{(1)} / 3\right) / w_{1}-M_{1133}^{(0) t}\right] / w_{2}+c_{0} q_{2}, \\
& y_{3}=\left[-\left(k^{(1)}-2 \mu^{(1)} / 3\right) / w_{1}-M_{3311}^{(0) t}\right] / w_{2}+c_{0} q_{3}, \\
& y_{4}=\left[\left(k^{(1)}+4 \mu^{(4)} / 3\right) / w_{1}-\left(M_{1111}^{(0) t}+M_{1122}^{(0)} t\right)\right] / w_{2}+c_{0} q_{4},
\end{aligned}
$$

where $w_{j}, q_{j}(j=1,2,3,4)$ are given in the appendix. The local tangent compliance tensors of the matrix $M_{i j k l}^{(0) t}$ can be obtained from Eq. (8):

$$
\begin{aligned}
& M_{1111}^{(0)}=M_{2222}^{(0)}=\frac{1}{9 k^{(0)}}+\frac{1}{3 \mu_{t}^{(0)}}+\frac{1}{9} \xi, \\
& M_{3333}^{(0)}=\frac{1}{9 k^{(0)}}+\frac{1}{3 \mu_{t}^{(0)}}+\frac{4}{9} \xi, \\
& M_{1122}^{(0)}=\frac{1}{9 k^{(0)}}-\frac{1}{6 \mu_{t}^{(0)}}+\frac{1}{9} \xi \\
& M_{1133}^{(0)}=\frac{1}{9 k^{(0)}}-\frac{1}{6 \mu_{t}^{(0)}}-\frac{2}{9} \xi, \\
& M_{3131}^{(0)}=\frac{1}{4 \mu_{t}^{(0)}}, \\
& M_{1212}^{(0)}=\frac{1}{4 \mu_{t}^{(0)}},
\end{aligned}
$$

where $\xi=1 / \lambda$.

To demonstrate the utility of the present incremental scheme, we illustrate two examples. In the first example, we consider a porous material. The material properties of the matrix are: $E^{(0)} / \sigma^{(0)}=200, v^{(0)}=0.30, n=0.10$, and the volume fraction of voids is $6.5 \%$. The constitutive 
response of the isotropic power-law hardening elastoplastic matrix can be characterized by the following strain potential:

$$
U^{(0)}(\boldsymbol{\sigma})=\frac{1}{2 k^{(0)}} \sigma_{m}^{2}+\frac{\sigma_{0}^{2}}{3 \mu^{(0)}}\left[\frac{n}{n+1}\left(\frac{\sigma_{e}}{\sigma_{0}}\right)^{n+1 / n}-\frac{1}{2}\left(\frac{\sigma_{e}}{\sigma_{0}}\right)^{2}\right] .
$$

In the initial calculation, we have used several sizes of the stress increment, i.e., $\Delta \Sigma=0.1,0.2,0.5$, $0.8 \mathrm{MPa}$. We find that the results are insensitive to the selected stress increment size. To speed up the calculation and obtain enough data of the calculated points, we finally select $\Delta \Sigma=0.5 \mathrm{MPa}$ in our formal calculations and this incremental size was also used in the following examples. Fig. 1 presents a comparison of the overall uniaxial stress-strain curve of the composite predicted by the present incremental method with that by the finite element method made by Hom and McMeeking [26]. The comparison shows that the result from the incremental method is slightly stiffer than that from the finite element method.

In the second example, a $\mathrm{SiC}_{\mathrm{p}} / 2124 \mathrm{Al}$ metal matrix composite material is investigated. The material properties of the phases are: $E^{(1)}=450 \mathrm{GPa}, v^{(1)}=0.17 ; \quad E^{(0)}=73 \mathrm{GPa}$, $v^{(0)}=0.33, \sigma_{0}=280 \mathrm{MPa}, n=7.66$ [27]. The volume fraction of the inclusion is taken to be 0.30 . The local strain potential of the isotropic power-law hardening elastoplastic metal matrix is given by

$$
U^{(0)}(\boldsymbol{\sigma})=\frac{1}{2 k^{(0)}} \sigma_{m}^{2}+\frac{1}{6 \mu^{(0)}} \sigma_{e}^{2}+\frac{n-1}{n+1} \frac{\sigma_{0}^{2}}{6 \mu^{(0)}}\left[1+\frac{2}{n+1}\left(\frac{\sigma_{e}}{\sigma_{0}}\right)^{n+1}\right] H\left(\sigma_{e}-\sigma_{0}\right),
$$

where $H(x)$ denotes Heaviside step function. The overall uniaxial stress-strain curve is calculated by the present incremental method and compared with those calculated by the classical secant

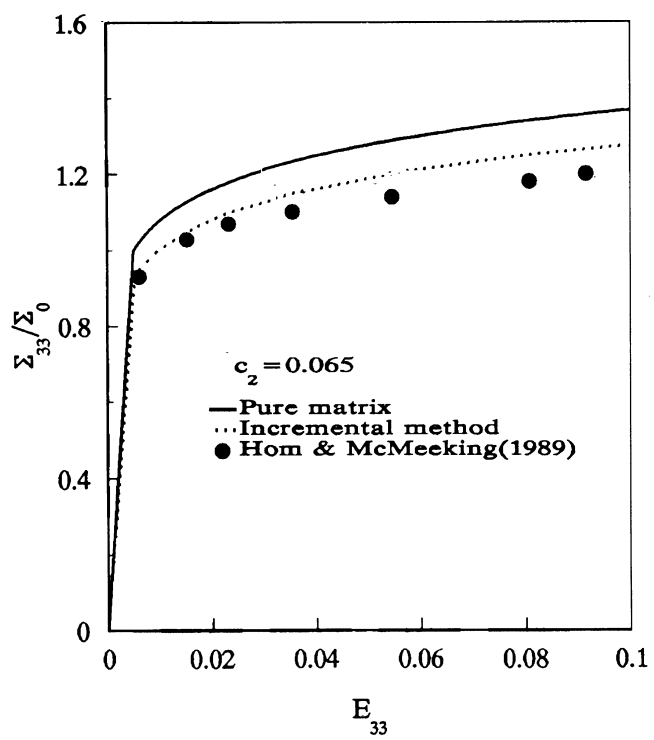

Fig. 1. Comparison of the stress-strain behavior of the incremental method with the finite element analysis for uniaxial tension $\left(\Sigma_{11}=\Sigma_{22}=0, \Sigma_{33}=\Sigma\right)$. 
modulus method (CSMM) and the modified secant modulus method (MSMM) (Fig. 2). It is seen from Fig. 2 that the solutions from the incremental method are much softer that those from CSMM and close to those from MSMM. For an axisymmetric tension case: $\Sigma_{11}=\Sigma_{22}=-0.5 \Sigma$, $\Sigma_{33}=\Sigma$, the tangent compliance tensors are also transversely isotropic. So, the overall stress-strain curves of this composite material can also be obtained (Fig. 3). From Fig. 3 we find

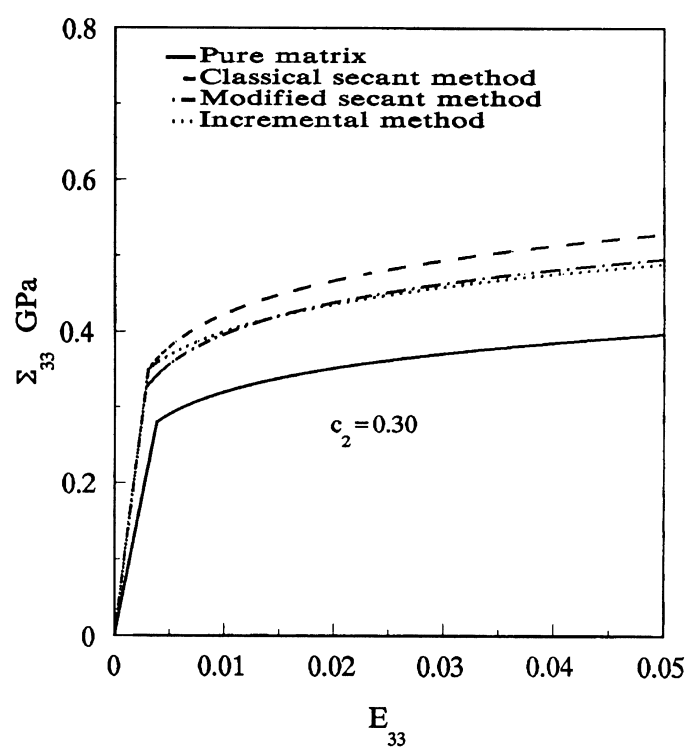

Fig. 2. Comparison of the stress-strain behavior of the incremental method with the classical secant modulus method and the modified secant modulus method for uniaxial tension $\left(\Sigma_{11}=\Sigma_{22}=0, \Sigma_{33}=\Sigma\right)$.

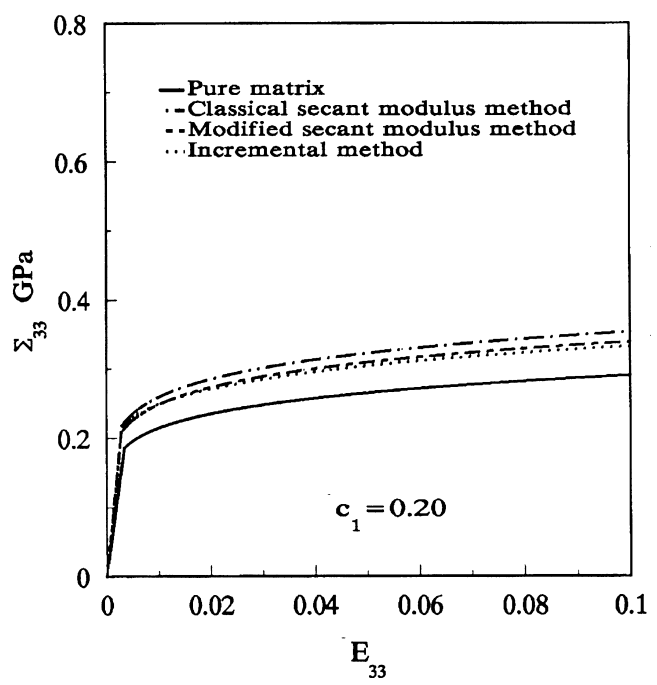

Fig. 3. Comparison of the stress-strain behavior of the incremental method with the classical secant modulus method and the modified secant modulus method for axisymmetric tension $\left(\Sigma_{11}=\Sigma_{22}=-0.5 \Sigma, \Sigma_{33}=\Sigma\right)$. 
that the result by the present incremental method is slightly softer than that by MSMM. Because the estimation of the effective properties of the associated linearized composites are all followed by the generalized self-consistent Mori-Tanaka model, the difference shown in Figs. 2 and 3 may be considered to result from the effect of the third stress invariant. Since the stress triaxiality in the present two examples is not high, the effect of the third stress invariant is not significant. These three examples demonstrate the utility of the present incremental scheme to characterize the overall behavior of nonlinear composites.

\section{Application to composites with debonding damage}

Under the influence of external mechanical load, stress concentrations develop at particle interfaces in particulate composites. This changes the initial two-phase composite, where all particles are bonded to the matrix into a three-phase composite where some particles become debonded, thereby creating voids. As theorized by many investigators, there are two prevailing criteria for void nucleation from the interfaces, namely, an energy condition and a stress condition [28]. Usually, the size of reinforcing particles in metal matrix composites (such as $\mathrm{SiCp} / \mathrm{Al}$ composites) is of the order of $10 \mu \mathrm{m}$. According to most of the previous studies, the energy criterion for void nucleation on this size is automatically satisfied [29]. That is, the stress criterion solely governs occurrence of the particles debonding (void nucleation). Apparently, the hard particles have not only a reinforcing effect but also a weakening effect due to particle debonding damage. Therefore, the overall mechanical behavior of the composites depends on the competitive result of these two effects. In this section, we will incorporate these two effects into the incremental formation presented in the previous section for predicting the overall behavior of particulate composites with debonding damage.

In order to describe the debonding process, as was done by Tohgo [11-13] in their research, the following assumptions are made: (1) the debonding of particles is controlled by the average hydrostatic stress of the particles and the statistical behavior of the particle-matrix interfacial strength. Here, the particle stress is used because the interfacial stress is given as a function of it [30]. Recent microscopic observations on the local deformation fields in $\mathrm{SiCp} / \mathrm{Al}$ composite made by Derrien et al. [31] have demonstrated that the matrix region adjacent to broken particles has high hydrostatic tension. This means that the hydrostatic stress is an important factor controlling particle debonding in particulate metal matrix composites. (2) Once the particles debond from the interfaces, these particles become voids; therefore the volume fraction of the debonded particles turns into a void volume fraction. Usually, the bonding strength of the interfaces is not uniform. Therefore, the debonding process can be described by probability theory. According to the experimental observations of Brechet et al. [32] and following the strategy adopted by Tohgo et al. [11-13], the cumulative probability of the debonding can be characterized by the Weibull distribution

$$
P_{v}\left(\bar{\sigma}_{m}^{(1)}\right)=1-\exp \left[-\left(\frac{\bar{\sigma}_{m}^{(1)}}{\beta}\right)^{\alpha}\right]
$$




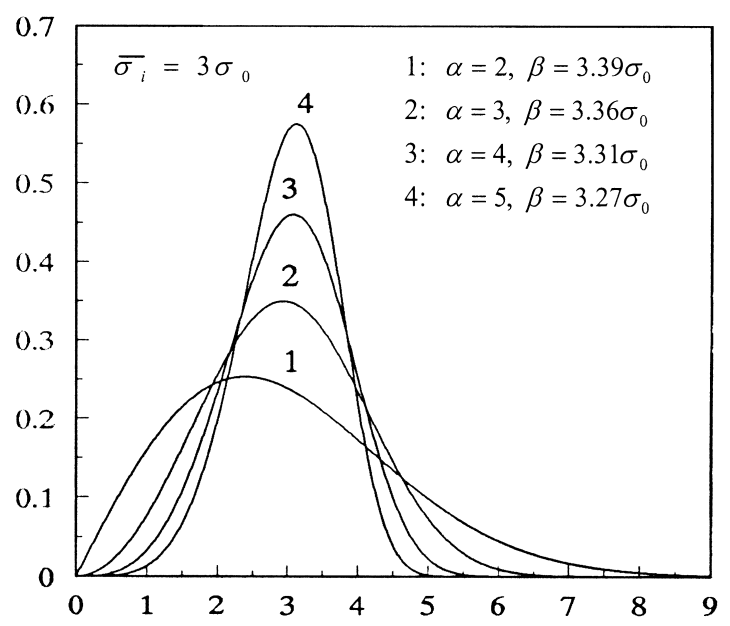

Fig. 4. Variations of the cumulative probability density of particle debonding with the distribution parameters $\alpha, \beta$.

where $\bar{\sigma}_{m}^{(1)}=\bar{\sigma}_{k k}^{(1)} / 3$ is the hydrostatic part of the stress of particles, and $\alpha$ and $\beta$ are the scale parameter and the shape parameter, respectively. This probability controls the volume fraction of the debonded particles or voids. With this function, the average interfacial strength of the interface, denoted by $\bar{\sigma}_{i}$, is related to the Gamma function $\Gamma(\cdot)$ as

$$
\bar{\sigma}_{i}=\beta \Gamma\left(1+\frac{1}{\alpha}\right)
$$

and the volume fraction of the debonded particles or voids $c_{2}$ is given by

$$
c_{2}=c_{p} P_{v}\left(\bar{\sigma}_{m}^{(1)}\right)=c_{p}\left\{1-\exp \left[-\left(\frac{\bar{\sigma}_{m}^{(1)}}{\beta}\right)^{\alpha}\right]\right\},
$$

where $c_{p}$ is the initial volume fraction of particles. $c_{p}$ is related to the volume fraction of the particles at the current state $c_{1}$ and the volume fraction of the debonded particles or voids $c_{2}$ as

$$
c_{1}+c_{2}=c_{p} .
$$

Fig. 4 presents the variations of the probability density of the debonding with the distribution parameters $\alpha$ and $\beta$.

Once particles debond from the interfaces, an initial particle/matrix two-phase composite turns into a particle/void/matrix three-phase hybrid composite. In this case, the overall tangent compliance tensor of the hybrid composite can be determined by the GSCMTM and written as

$$
\overline{\mathbf{M}}_{t}=\left[c_{0} \stackrel{(4)}{\mathbf{I}}+c_{1} \mathbf{H}^{(1)^{-1}}+c_{2} \mathbf{H}^{(2)^{-1}}\right]: M_{\mathbf{t}}^{(0)}:\left[c_{0} \stackrel{(4)}{\mathbf{I}}+c_{1} \mathbf{T}^{(1)^{-1}}\right]^{-1},
$$


where

$$
\mathbf{T}^{(1)}=\stackrel{(4)}{\mathbf{I}}+\mathbf{Q}:\left(\mathbf{M}^{(1)}-\mathbf{M}^{(0)}\right)
$$

and

$$
\mathbf{Q}=\mathbf{M}^{(0)^{-1}}-\mathbf{M}^{(0)^{-1}}: \mathbf{P}: \mathbf{M}^{(0)^{-1}}
$$

in which $\mathbf{P}=\mathbf{S}: \mathbf{M}^{(0)}$, and $\mathbf{S}$ is the inclusion Eshelby tensor.

Since there is only one independent variable among $\Sigma, c_{1}$ and $c_{2}$, the overall incremental constitutive relation of the three-phase hybrid composite can still be written in the form of Eq. (11). The effect of the damage on the overall behavior of the composite is included in the scheme by recalculating the values of $c_{1}$ and $c_{2}$ at each incremental step according to the assumed damage evolution law given by Eq. (27). Following a procedure similar to that presented in the previous section, the overall stress-strain curves of nonlinear composite with progressive debonding damage can be obtained. Here, we only consider the uniaxial tension case. An initially perfectly bonded hard elastic particle-reinforced soft elastic-plastic metal matrix composite is investigated. The elastic constants of the phases are given respectively as $E^{(1)}=5000 \sigma_{0}, v(1)=0.17 ; E^{(0)}=500 \sigma_{0}$, $v^{(0)}=0.30$. The constitutive behavior of the isotropic power-law hardening elastoplastic matrix is characterized by the following strain potential:

$$
U(\boldsymbol{\sigma})=\frac{1}{2 k^{(0)}} \sigma_{m}^{2}+\frac{\sigma_{0}^{2}}{E^{(0)}}\left[\frac{1}{n+1}\left(\frac{\sigma_{e}}{\sigma_{0}}\right)^{n+1}+\frac{2\left(1+v^{(0)}\right)}{3}\left(\frac{\sigma_{e}}{\sigma_{0}}\right)^{2}-\left(\frac{\sigma_{e}}{\sigma_{0}}\right)\right],
$$

where $\sigma_{0}$ is the yield stress of the matrix, and material constant $n=10$. The cumulative probability of particles debonding is given by Eq. (25) and the distribution parameters are, respectively, taken as $\beta=3.27 \sigma_{0}, \alpha=5$. According to Eq. (26), this set of parameters gives the average interfacial strength $\bar{\sigma}_{i}=3 \sigma_{0}$. Figs. 5a-d present the overall uniaxial stress-strain curves for the initial particle volume fraction $c_{1}=0.05,0.10,0.20,0.30$, respectively. From Fig. 5 we find that particle debonding has a significant weakening effect on the overall behavior of the composite. For the case of $c_{1}=0.20$, when the overall strain approaches 0.1 , the hard particle reinforcing effect is almost counteracted by the particle debonding. Therefore, the particle debonding weakening effect should be considered when this class of hard-particle reinforced composites is applied in engineering fields.

\section{Conclusions}

Based on the generalized self-consistent Mori-Tanaka method, a general incremental micromechanical scheme is developed in this paper. The advantage of the scheme is that it can reflect, at least partly, the effects of the third invariant of the stress. Comparison with the other existing theoretical and numerical results demonstrates that the present incremental scheme is very satisfactory. By making use of this scheme, the effects of the progressive particle debonding damage on the overall behavior of nonlinear composites are investigated. The results show that the weakening effects of particle debonding should be considered when hard-particle reinforced composites are used in engineering fields. 


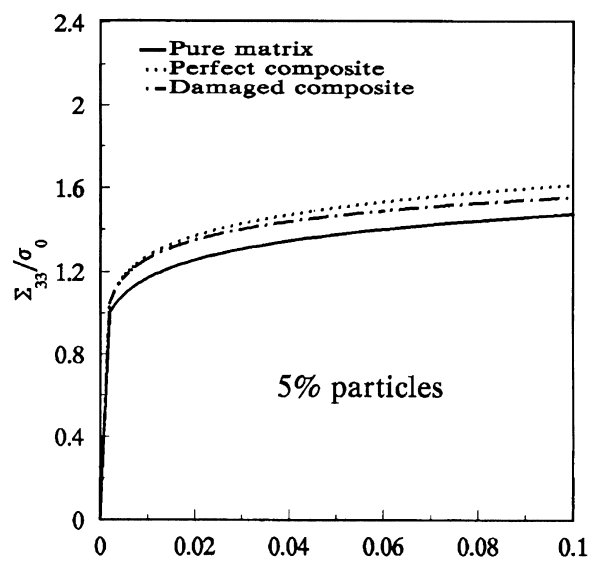

(a)

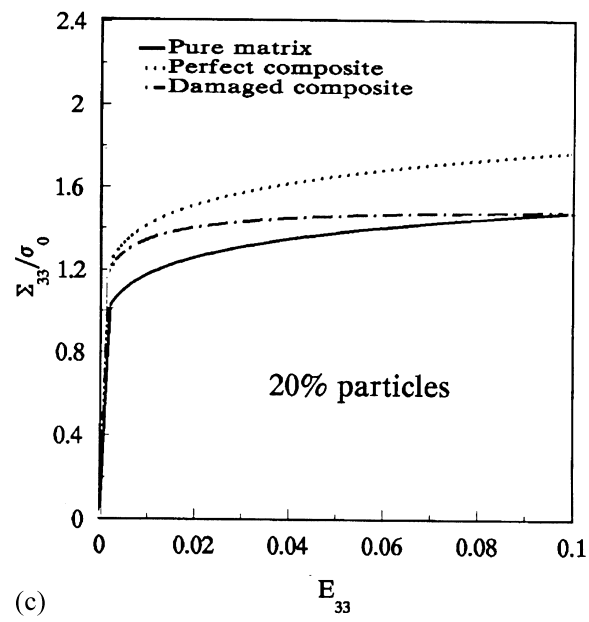

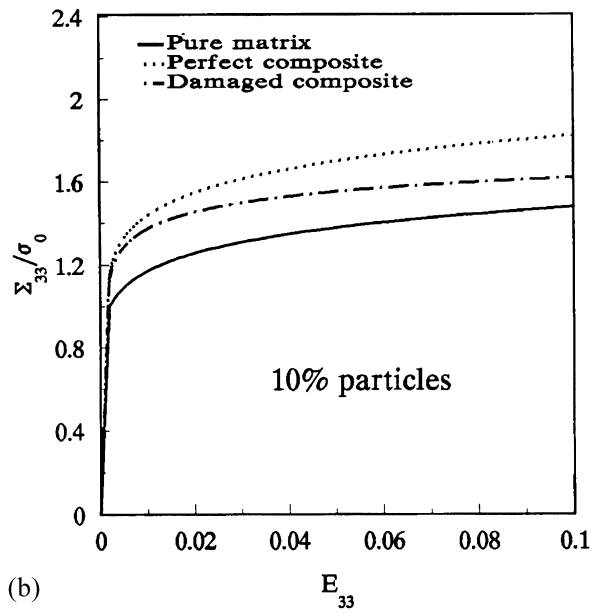

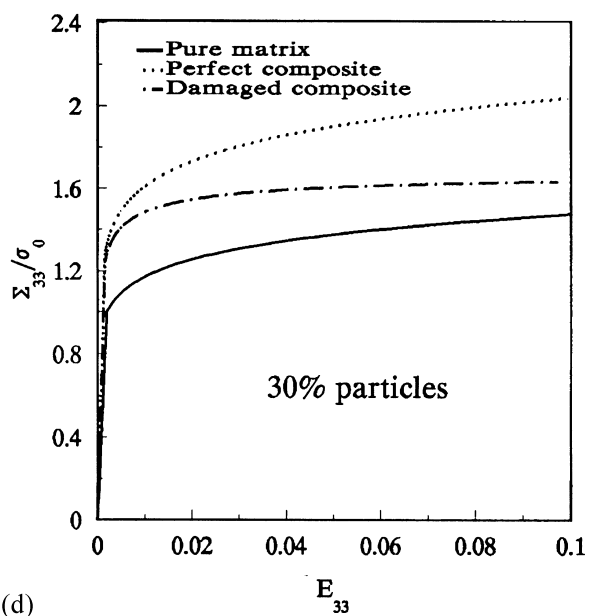

Fig. 5. Stress-strain behavior of pure matrix material, perfect composite, and damage composite under uniaxial tension $\left(\Sigma_{11}=\Sigma_{22}=0, \Sigma_{33}=\Sigma\right):$ (a) $c_{p}=5 \%$; (b) $c_{p}=10 \%$; (c) $c_{p}=20 \%$; (d) $c_{p}=30 \%$.

\section{Acknowledgements}

The authors gratefully acknowledge the financial support of this work by the National Natural Science Foundation of China (19902017 and 19891180) and Major Project of Chinese Academy of Sciences (KJ-951-1-201)

\section{Appendix. The related parameters in Eq. (20)}

The related parameters used in Eq. (20) are given, respectively, as follows:

$$
y_{1}=\left[2\left(k^{(1)}+\mu^{(1)} / 3\right) / w_{1}-M_{3333}^{(0)}\right] / w_{2}+c_{0} q_{1},
$$




$$
\begin{aligned}
& y_{2}=\left[-\left(k^{(1)}-2 \mu^{(1)} / 3\right) / w_{1}-M_{1133}^{(0)}\right] / w_{2}+c_{0} q_{2}, \\
& y_{3}=\left[-\left(k^{(1)}-2 \mu^{(1)} / 3\right) / w_{1}-M_{3311}^{(0)}\right] / w_{2}+c_{0} q_{3}, \\
& y_{4}=\left[\left(k^{(1)}+4 \mu^{(4)} / 3\right) / w_{1}-\left(M_{1111}^{(0)}+M_{1122}^{(0)}\right)\right] / w_{2}+c_{0} q_{4},
\end{aligned}
$$

where

$$
\begin{aligned}
w_{1}= & 2\left(k^{(1)}+4 \mu^{(1)} / 3\right)\left(k^{(1)}+\mu^{(1)} / 3\right)-2\left(k^{(1)}-2 \mu^{(1)} / 3\right)^{2}, \\
w_{2}= & {\left[2\left(k^{(1)}+\mu^{(1)} / 3\right) / w_{1}-M_{3333}^{(0)}\right]\left[\left(k^{(1)}+4 \mu^{(4)} / 3\right) / w_{1}-\left(M_{111}^{(0)}+M_{1122}^{(0)}\right)\right] } \\
& -2\left[-\left(k^{(1)}-2 \mu^{(1)} / 3\right) / w_{1}-M_{1133}^{(0)}\right]\left[-\left(k^{(1)}-2 \mu^{(1)} / 3\right) / w_{1}-M_{3311}^{(0)}\right]
\end{aligned}
$$

and

$$
\begin{aligned}
& q_{1}=r_{1}-a_{1}, \quad q_{2}=r_{2}-a_{2}, \\
& q_{3}=r_{3}-a_{3}, \quad q_{4}=r_{4}-a_{4},
\end{aligned}
$$

where

$$
\begin{aligned}
& r_{1}=M_{3333}^{(0)} /\left[\left(M_{1111}^{(0)}+M_{1122}^{(0)}\right) M_{3333}^{(0)}-2 M_{1133}^{(0)} M_{3311}^{(0)}\right], \\
& r_{2}=-M_{1133}^{(0)} /\left[\left(M_{1111}^{(0)}+M_{1122}^{(0)}\right) M_{3333}^{(0)}-2 M_{1133}^{(0)} M_{3311}^{(0)}\right], \\
& \left.r_{3}=-M_{3311}^{(0)} /\left[\left(M_{1111}^{(0)}+M_{1122}^{(0)}\right) M_{3333}^{(0)}-2 M_{1133}^{(0)} M_{3311}^{(0)}\right], \quad{ }_{3311}\right] \\
& r_{4}=\left(M_{1111}^{(0)}+M_{1122}^{(0)}\right) /\left[\left(M_{1111}^{(0)}+M_{1122}^{(0)}\right) M_{3333}^{(0)}-2 M_{1133}^{(0)} M\right.
\end{aligned}
$$

and

$$
\begin{aligned}
& a_{1}=r_{1}\left(p_{1} r_{1}+2 p_{3} r_{2}\right)+2 r_{3}\left(p_{2} r_{1}+p_{4} r_{2}\right), \\
& a_{2}=r_{2}\left(p_{1} r_{1}+2 p_{3} r_{2}\right)+r_{4}\left(p_{2} r_{1}+p_{4} r_{2}\right), \\
& a_{3}=r_{3}\left(p_{4} r_{4}+2 p_{2} r_{3}\right)+r_{1}\left(p_{3} r_{4}+p_{1} r_{3}\right), \\
& a_{4}=r_{4}\left(p_{4} r_{4}+2 p_{2} r_{3}\right)+2 r_{2}\left(p_{3} r_{4}+p_{1} r_{3}\right),
\end{aligned}
$$

where

$$
\begin{aligned}
& p_{1}=\frac{1}{4 \pi}\left(\bar{G}_{1111}+\bar{G}_{1212}\right), \\
& p_{2}=\frac{1}{4 \pi} \bar{G}_{3131}, \\
& p_{2}=\frac{1}{4 \pi} \bar{G}_{1313}, \\
& p_{4}=\frac{1}{4 \pi} \bar{G}_{3333} .
\end{aligned}
$$


Here $\bar{G}_{i j k l}$ are written as

$$
\begin{aligned}
\bar{G}_{1111}= & \bar{G}_{2222}=\frac{\pi}{2} \int_{0}^{1} \omega\left(1-x^{2}\right)\left\{\left[f\left(1-x^{2}\right)+h \rho^{2} x^{2}\right]\left[(3 e+d)\left(1-x^{2}\right)+4 f \rho^{2} x^{2}\right]\right. \\
& \left.-g^{2} \rho^{2} x^{2}\left(1-x^{2}\right)\right\} \mathrm{d} x, \\
\bar{G}_{3333}= & 4 \pi \int_{0}^{1} \omega \rho^{2} x^{2}\left[d\left(1-x^{2}\right)+f \rho^{2} x^{2}\right]\left[e\left(1-x^{2}\right)+f \rho^{2} x^{2}\right] \mathrm{d} x, \\
\bar{G}_{1122}= & \frac{\pi}{2} \int_{0}^{1} \omega\left(1-x^{2}\right)\left\{\left[f\left(1-x^{2}\right)+h \rho^{2} x^{2}\right]\left[(e+3 d)\left(1-x^{2}\right)+4 f \rho^{2} x^{2}\right]\right. \\
& \left.-3 g^{2} \rho^{2} x^{2}\left(1-x^{2}\right)\right\} \mathrm{d} x, \\
\bar{G}_{1133}= & \bar{G}_{2233}=2 \pi \int_{0}^{1} \omega \rho^{2} x^{2}\left\{\left[(d+e)\left(1-x^{2}\right)+2 f \rho^{2} x^{2}\right]\left[f\left(1-x^{2}\right)+h \rho^{2} x^{2}\right]\right. \\
& \left.-g^{2} \rho^{2} x^{2}\left(1-x^{2}\right)\right\} \mathrm{d} x, \\
\bar{G}_{3311}= & \bar{G}_{3322}=2 \pi \int_{0}^{1} \omega\left(1-x^{2}\right)\left[d\left(1-x^{2}\right)+f \rho^{2} x^{2}\right]\left[e\left(1-x^{2}\right)+f \rho^{2} x^{2}\right] \mathrm{d} x, \\
\bar{G}_{1212}= & \frac{\pi}{2} \int_{0}^{1} \omega\left(1-x^{2}\right)^{2}\left\{g^{2} \rho^{2} x^{2}-(d-e)\left[f\left(1-x^{2}\right)+h \rho^{2} x^{2}\right]\right\} \mathrm{d} x, \\
\bar{G}_{11313}= & \left.\bar{G}_{2323}=-(2 \pi) \int_{0}^{1} \omega g \rho^{2} x^{2}\left(1-x^{2}\right)\left[e\left(1-x^{2}\right)+f \rho^{2} x^{2}\right]\right\} \mathrm{d} x,
\end{aligned}
$$

where

$$
\omega^{-1}=\left[e\left(1-x^{2}\right)+f \rho^{2} x^{2}\right]\left\{\left[d\left(1-x^{2}\right)+f \rho^{2} x^{2}\right]\left[f\left(1-x^{2}\right)+h \rho^{2} x^{2}\right]-g^{2} \rho^{2} x^{2}\left(1-x^{2}\right)\right\},
$$

in which $\rho$ is the aspect ratio of the inclusion.

\section{References}

[1] Accorsi ML, Nemat-Nasser S. Bounds on the overall elastic and instantaneous elastoplastic moduli of periodic composites. Mechanics of Materials 1986;5:209-20.

[2] Tandon GP, Weng GJ. A theory of particle-reinforced plasticity. Journal of Applied Mechanics 1988;55:126-35.

[3] Berveiller M, Zaoui A. An extension of the self-consistent scheme to plastically-flowing polycrystals. Journal of Mechanics and Physics of Solids 1979;26:325-44.

[4] Weng GJ. A unified self-consistent theory for plastic-creep deformation of metals. Journal of Applied Mechanics 1982;49:728-34.

[5] Qiu YP, Weng GJ. A theory of plasticity for porous materials and particle-reinforced composites. Journal of Applied Mechanics 1992;59:261-8.

[6] Qiu YP, Weng GJ. An energy approach to the plasticity of two-phase composite containing alifned inclusions. Journal of Applied Mechanics 1995;62:1039-46.

[7] $\mathrm{Hu}$ G. A method of plasticity for general aligned spheroidal void or fiber reinforced composites. International Journal of Plasticity 1996;12:439. 
[8] Ju JW, Tseng KH. Effective elastoplastic behavior of two-phase ductile matrix composites. International Journal of Solids Structures 1996;33:4257-91.

[9] Ponte Castaneda P. The effective mechanical properties of nonlinear isotropic composites. Journal of Mechanics Physics of Solids 1991;39:45-71.

[10] Ponte Castaneda P. New variational principles in plasticity and their applications to composite materials. Journal of Mechanics and Physics of Solids 1992;40:1757-88.

[11] Tohgo K, Weng GJ. A progressive damage mechanics in particle-reinforced metal matrix composites under high triaxial tension. ASME Journal of Engineering Materials Technology 1994;116:414-23.

[12] Tohko K, Chou T-W. Incremental theory of particulate-reinforced composites including damage. JSME International Journal, Series A 1996;39(3):389-97.

[13] Tohko K, Mochizuki M, Ishii H. Incremental damage theory and its application to glass-particle-reinforced Nylon 66 composites. International Journal of Mechanical Sciences 1998;40:199-213.

[14] Eshelby JD. The determination of the elastic field of an elliposidal inclusion and related problem. Proceedings of Royal Society of London 1957;A241:376-96.

[15] Mori T, Tanaka K. Average stress in matrix and average elastic energy of materials with misfitting inclusions. Acta Metallurgica 1973;21:571-4.

[16] Lee B, Mear WE. Effect of inclusion shape on the stiffness of nonlinear two-phase composites. Journal of Mechanics and Physics of Solids 1991;39:627-49.

[17] Dai LH, Huang ZP, Wang R. A generalized self-consistent Mori-Tanaka scheme for the prediction of the effective moduli of hybrid multiphase particulate composites. Polymer Composites 1998;19:506-13.

[18] Dai LH, Huang ZP, Wang R. A generalized self-consistent Mori-Tanaka model and the effective moduli of coated inclusion based composites. Acta Mechanica Solida 1998;18:199-208.

[19] Christensen RM, Lo KH. Solutions for effective shear properties of three-phase sphere and cylinder models. Journal of Mechanics and Physics of Solids 1979;27:315-30.

[20] Dederichs PH, Zeller R. Variational treatment of the elastic constants of disorder materials. Z. Physics 1973;259:103-16.

[21] Hill R. Interfacial operators in the mechanics of composite media. Journal of Mechanics and Physics of Solids 1983;31:347-57.

[22] Dai LH. Effective elastoplastic theory of multiphase particulate composites. Peking University Post-doctoral Research Report, Peking University, 1998.

[23] Weng GJ. Some elastic properties of reinforced solids with special reference to isotropic ones containing spherical inclusions. International Journal of Engineering Science 1984;22:845-56.

[24] Mura T. Micromechanics of defects in solids. The Hague: Nijhoff, 1987.

[25] Walpole LJ. On the overall elastic moduli of composite materials. Journal of Mechanics and Physics of Solids 1969;17:235-51.

[26] Hom CL, McMeeking RM. Void growth in elastic-plastic materials. Journal of Applied Mechanics 1989;56:309-17.

[27] Li G, Ponte Castaneda P. Variational estimates for the elastoplastic response of particle-reinforced metal-matrix composites. Applied Mechanics Review 1994;47:s77-94.

[28] Fisher JK, Gurand J. Void nucleation in spheroidized carbon steels. Metallurgical Science 1981;15:185-202.

[29] Argon AS. Cavity formation from inclusion in ductile fracture. Metallurgical Transactions 1975;6:839-51.

[30] Tandon GP, Weng GJ. Stress distribution in and around spheroidal inclusions and voids at finite concentration. ASME Journal of Applied Mechanics 1986;53:511-7.

[31] Derrien $\mathrm{K}$ et al. Multiscale modeling of the damaged plastic behavior and failure of $\mathrm{Al} / \mathrm{SiCp}$ composites. International Journal of Plasticity 1999;15:667-85.

[32] Brechet Y, Embury JD, Tao S, Luo L. Damage initiation in metal matrix composites. Acta Metallurgical Materialia 1991;39:1781-9. 